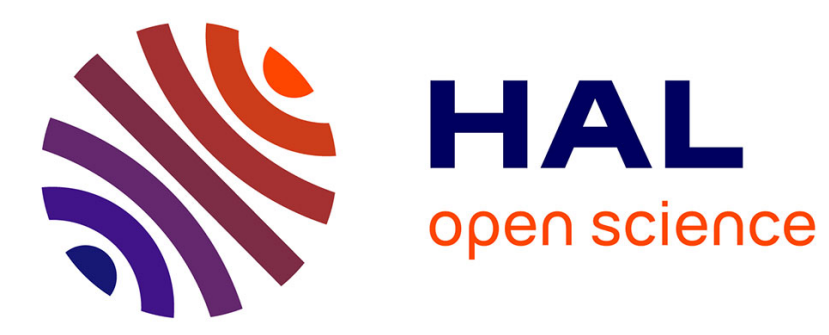

\title{
Adiabatic spectrum for relativistic hydrogen in a strong homogeneous magnetic field
}

Pierre Duclos, Philippe Briet, Raymond Brummelhuis

\section{To cite this version:}

Pierre Duclos, Philippe Briet, Raymond Brummelhuis. Adiabatic spectrum for relativistic hydrogen in a strong homogeneous magnetic field. Fifth Workshop on Critical Stability „Oct 2008, Erice, Italy. pp.179-182, 10.1007/s00601-009-0014-y . hal-00368395

\section{HAL Id: hal-00368395 https://hal.science/hal-00368395}

Submitted on 16 Mar 2009

HAL is a multi-disciplinary open access archive for the deposit and dissemination of scientific research documents, whether they are published or not. The documents may come from teaching and research institutions in France or abroad, or from public or private research centers.
L'archive ouverte pluridisciplinaire HAL, est destinée au dépôt et à la diffusion de documents scientifiques de niveau recherche, publiés ou non, émanant des établissements d'enseignement et de recherche français ou étrangers, des laboratoires publics ou privés. 


\title{
Adiabatic spectrum for relativistic hydrogen in a strong homogeneous magnetic field
}

\author{
R. Brummelhuis ${ }^{1,2 *}$, Ph. Briet ${ }^{3 * *}$, P. Duclos ${ }^{3 * * *}$
}

${ }^{1}$ Université de Reims, FR 3111, Département de Mathématique et Informatique, BP 1039, 51687 Reims Cedex 2, France

${ }^{2}$ Birkbeck, University of London, School of Economics, Mathematics and Statistics, Malet Street, N1PB, London, UK

${ }^{3}$ Centre de Physique Théorique de Marseille UMR 6207, Unité Mixte de Recherche du CNRS et des Universités Aix-Marseille I, Aix-Marseille II et de l'Université du Sud Toulon-Var, Laboratoire affilié á la FRUMAM, Luminy Case 907, 13288 Marseille Cedex 9, France

\begin{abstract}
We study the bound states of relativistic hydrogen-like atoms coupled to strong homogeneous magnetic fields, assuming a fixed, infinitely heavy nucleus. Working in the adiabatic approximation in which the electron is confined to the lowest Landau level, we show that the corresponding Dirac Hamiltonian always has an infinite discrete spectrum accumulating at $m c^{2}$, $m$ being the electron mass, and that, as the field strength increases, its eigenvalues successively descend into the lower part of the continuous spectrum, $\left(-\infty,-m c^{2}\right]$. This phenomenon is for large $B$ roughly periodical in $\log \mathrm{B}$.
\end{abstract}

\section{Introduction}

The Dirac Hamiltonian for a hydrogen-like atom with nuclear charge $Z$ in a constant magnetic field $\mathbb{B}$ of size $B$ in the $z$-direction is given by

$$
D^{B}=D_{0}^{B}-\frac{\gamma}{|r|}, \quad D_{0}^{B}=\vec{\alpha} \cdot(p+\mathbb{A})+\beta
$$

where we use coordinates $r=(x, y, z) \in \mathbb{R}^{3}$ and where $p=i^{-1} \nabla_{r}$. Furthermore, $\gamma:=\alpha Z$ with $\alpha$ the fine structure constant, $\beta$ and $\vec{\alpha}=\left(\alpha_{x}, \alpha_{y}, \alpha_{z}\right)$ are the Dirac-matrices:

$$
\alpha_{j}=\left(\begin{array}{cc}
0 & \sigma_{j} \\
\sigma_{j} & 0
\end{array}\right)(j=x, y, z), \quad \beta=\left(\begin{array}{cc}
I & 0 \\
0 & -I
\end{array}\right),
$$

with $\sigma_{j}$ the well-known Pauli-matrices, $I$ is the $2 \times 2$-identity matrix, and $\mathbb{A}$ is the vector potential, which we choose as $\mathbb{A}:=\frac{1}{2} \mathbb{B} \wedge r=\frac{1}{2} B(-y, x, 0)$.

\footnotetext{
*E-mail address: raymond.brummelhuis@univ-reims.fr

**E-mail address: briet@univ-tln.fr

***E-mail address: duclos@univ-tln.fr
} 


\subsection{Spectral decomposition of $D_{0}^{B}$}

Since $\mathbb{A}$ has a component 0 in the $z$-direction, we can decompose $D_{0}^{B}$ into a transversal and a parallel operator with respect to the magnetic field: $D_{0}^{B}=$ $D_{0, \mathrm{tr}}^{B}+D_{0, / /}$, where $D_{0, / /}:=\alpha_{z} p_{z}+\beta m$ is independent of $B$, and where $D_{0, \mathrm{tr}}^{B}:=$ $\alpha_{x}\left(p_{x}-\frac{1}{2} B y\right)+\alpha_{y}\left(p_{y}+\frac{1}{2} B x\right)$. One has $\left(D_{0, \mathrm{tr}}^{B}\right)^{2}=H^{B} \otimes I$, where $H^{B}=\left(p_{x}-\right.$ $\left.\mathbb{A}_{x}\right)^{2}+\left(p_{y}-\mathbb{A}_{y}\right)^{2}+B \sigma_{z}=-\Delta_{x, y}+\frac{1}{4} B\left(x^{2}+y^{2}\right)+B L_{z}+B \sigma_{z}$ is the PauliHamiltonian in a constant magnetic field, which has an explicitly known pure point-spectrum, consisting of non-negative integer multiples of $2 B$, each of which is has infinite multiplicity (cf. e.g. [2]). Let $\Pi_{\mathcal{L}}^{B}$ be the orthogonal projection onto the (infinite dimensional) kernel of $\left(D_{0, \mathrm{tr}}^{B}\right)^{2}$. Since for a self-adjoint operator $A$, $\operatorname{Ker}\left(A^{2}\right)=\operatorname{Ker}(A)$, this is also the orthogonal projection onto the kernel of $D_{0, \mathrm{tr}}^{B}$, which we will call the relativistic lowest Landau-level, $\mathcal{L}^{B}$, of $D_{0, \mathrm{tr}}^{B}$; here "lowest" has to be interpreted in the sense of absolute value: the spectrum of $D_{0, \mathrm{tr}}^{B}$ can be shown to be $\sqrt{2 B} \mathbb{Z}$, and consequently $\left|D_{0, \mathrm{tr}}^{B}\right| \geq \sqrt{2 B}$ on the orthogonal complement of $\mathcal{L}^{B}$.

To remove the infinite degeneracy, we take advantage of the fact that $D^{B}$, $D_{0}^{B}$ and $D_{0, \mathrm{tr}}^{B}$ all commute with $J_{z}=L_{z}+S_{z}$, the total angular momentum in the $z$-direction, and that we can therefore fix an angular momentum channel $J_{z}=j, j \in \frac{1}{2}+\mathbb{Z}$. If we indicate restrictions to this angular momentum channel by the superscript " $J_{z}=j$ ", then we have that the image of $\Pi_{\mathcal{L}}^{B, J_{z}=j}$ is non-zero iff $j=\ell-1 / 2$ with $\ell \leq 0$. Moreover, for such $j, \operatorname{Im} \Pi_{\mathcal{L}}^{B, J_{z}=j}$ is one-dimensional and spanned by the spinor

$$
\chi_{\ell}(x+i y)\left(\begin{array}{l}
0 \\
1 \\
0 \\
1
\end{array}\right), \quad \chi_{\ell}\left(\rho e^{i \varphi}\right):=\left(2 \pi 2^{\ell} \ell !\right)^{-1 / 2} B^{1 / 2} \rho^{\ell} e^{-i \ell \varphi} e^{-B \rho^{2} / 4}
$$

We still have that $\left|D_{0}^{B, J_{z}=j}\right| \geq \sqrt{2 B}$ on the orthogonal complement of $\operatorname{Im} \Pi_{\mathcal{L}}^{B, J_{z}=\ell}$. For simplicity we will limit ourselves to the case of $j=-1 / 2$. This is not an essential restriction, though, and our calculations will carry through, with modifications, for general $j \in-\frac{1}{2}-\mathbb{N}$.

\section{Adiabatic approximation}

We will work in the adiabatic approximation, in which, for large $B$, the electron is assumed to be "frozen" in its lowest Landau orbits in directions perpendicular to the field $\mathbb{B}$. This means replacing the exact Hamiltonian $D^{B}$ by the "lowest Landau" Hamiltonian $d_{\mathcal{L}}^{B}:=\Pi_{\mathcal{L}}^{B, J_{z}=-1 / 2} D^{B} \Pi_{\mathcal{L}}^{B, J_{z}=-1 / 2}$ (where we henceforth leave off the superscript indicating the angular momentum channel). The Hamiltonian $d_{\mathcal{L}}^{B}$ is one-dimensional, and effectively acts on two-component wave-functions as

$$
d_{\mathcal{L}}^{B}=d_{0, z}+V_{\mathcal{L}}^{B}(z), d_{0, z}=\left(\begin{array}{cc}
m & p_{z} \\
p_{z} & -m
\end{array}\right)
$$


with $V_{\mathcal{L}}^{B}(z):=-\gamma\left\langle\left.\chi_{0}^{B}|| r\right|^{-1} \mid \chi_{0}^{B}\right\rangle=\sqrt{B} V_{\mathcal{L}}^{1}(\sqrt{B} z) ;$ explicitly,

$$
V_{\mathcal{L}}^{1}(z)=-\gamma \int_{0}^{\infty} \frac{e^{-u}}{\sqrt{2 u+z^{2}}} d u .
$$

Note that $d_{0}^{B}$ is the free Dirac operator in dimension 1.

This of course begs the question of whether we can relate the spectra of $D^{B}$ and $d_{\mathcal{L}}^{B}$. For large $B$, a large amount of (positive or negative) energy is needed to go from the lowest Landau level into one of the others, and the question is whether the attractive Coulomb potential can provide that energy. In the nonrelativistic case the answer was "no", at least asymptotically for very large $B$; mathematically, this translated into the asymptotic equality, in norm resolvent sense, of the full Hamiltonian and its projection onto the lowest Landau level in [2]. In the relativistic case, the situation is not that clear, basically because both Coulomb potential and the Dirac operator have the same order of homogeneity -1 , a well-known problem in rigorous relativistic quantum mechanics. A further analysis, using the techniques of [2], shows that one can prove norm-resolvent convergence of $D^{B}$ to $d_{\mathcal{L}}^{B}$ if we allow $\gamma$ to be $B$-dependent and require that $\gamma \sqrt{B} \rightarrow 0$, cf. [1]. More generally, we can do perturbation theory around $d_{\mathcal{L}}^{B}$ if $\gamma \sqrt{B} \ll 1$, that is $B \ll \alpha^{2} \simeq 18769$ for hydrogen. Since in our units $B=1$ already corresponds to $4.410^{9}$ tesla, this may not be an unreasonable assumption for the lighter atoms.

\section{Large- $B$ approximation of $d_{\mathcal{L}}^{B}$}

The eigenvalue-problem for $d_{\mathcal{L}}^{B}$ does not seem to be directly solvable in closed form, but we can further simplify the operator for large values of $B$. If $U_{\pi / 2}$ denotes the rotation of $\mathbb{C}^{2}$ by $\pi / 4$, it is convenient to introduce

$$
\widetilde{d}_{\mathcal{L}}^{B}:=U_{\pi / 4}^{-1} d_{\mathcal{L}}^{B} U_{\pi / 4}=\left(\begin{array}{cc}
p_{z}+V_{\mathcal{L}}^{B} & -m \\
-m & -p_{z}+V_{\mathcal{L}}^{B}
\end{array}\right)
$$

We note that for $z \neq 0$ and as $B \rightarrow \infty, V_{\mathcal{L}}^{B}(z) \rightarrow-\gamma|z|^{-1}$. One can now show that as $B \rightarrow \infty, \widetilde{d}_{\mathcal{L}}^{\infty, B}$ is asymptotic, in norm-resolvent sense, to a suitably defined regularization of the one-dimensional Dirac + Coulomb Hamiltonian (rotated by $\pi / 4)$ :

Proposition 3.1 Define the operator $d_{\mathcal{L}}^{\infty, B}$ on $L^{2}\left(\mathbb{R}, \mathbb{C}^{2}\right)$ by

$$
\widetilde{d}_{\mathcal{L}}^{\infty, B}:=\left(\begin{array}{cc}
p_{z}-\gamma /|z| & m \\
m & -p_{z}-\gamma /|z|
\end{array}\right),
$$

with domain those $u=\left(u_{1}, u_{2}\right) \in L^{2}\left(\mathbb{R}, \mathbb{C}^{2}\right)$ such that $u_{j} \in H^{1}(|z| \geq \varepsilon)$ for all $\varepsilon>0(j=1,2)$ and satisfying the following boundary condition in 0 :

$$
e^{i(-1)^{j} \gamma(\log B+c)} \varepsilon^{i(-1)^{j} \gamma} u_{j}(\varepsilon) \simeq \varepsilon^{-i(-1)^{j} \gamma} u_{j}(-\varepsilon), \quad \varepsilon \downarrow 0, \quad j=1,2,
$$

where $c=\log 2-\Gamma^{\prime}(1)$, and $\simeq$ means that the difference tends to 0 with $\varepsilon$. Then $d_{\mathcal{L}}^{\infty, B}$ is self-adjoint and we have that for all complex $\xi \notin \mathbb{R}$,

$$
\lim _{B \rightarrow \infty}\left\|\left(\widetilde{d}_{\mathcal{L}}^{B}-\xi\right)^{-1}-\left(\widetilde{d}_{\mathcal{L}}^{\infty, B}-\xi\right)^{-1}\right\|=0
$$


Observe that $\widetilde{d}_{\mathcal{L}}^{\infty, B}$ still depends on $B$, through the boundary conditions, which in fact are periodic in $\log B$. The eigenvalue problem for $\widetilde{d}_{\mathcal{L}}^{\infty, B}$ can be explicitly solved with the help of Whittaker functions. Before stating the result, we note the following symmetry of the operator: let $P: u(z) \rightarrow u(-z)$ be the parity operator, and let $\mathcal{E}:\left(u_{1}, u_{2}\right) \rightarrow\left(u_{2}, u_{1}\right)$ exchange the components of the spinor $u$. Then $\widetilde{d}_{\mathcal{L}}^{\infty, B}$ commutes with $P \mathcal{E}$, which has eigenvalues \pm 1 , and we can reduce the operator accordingly.

Theorem 3.2 Let $F_{ \pm}:(-m, m) \rightarrow\{\zeta \in \mathbb{C}:|\zeta|=1\}$ be given by

$$
F_{ \pm}(E):=(\mp) \frac{E+i \tau / 2}{|E+i \tau / 2|} \cdot \frac{\Gamma(1-2 i \gamma)}{\Gamma(1+2 i \gamma)} \cdot \frac{\Gamma(1+i \gamma-\kappa)}{\Gamma(1-i \gamma-\kappa)} \cdot \tau^{2 i \gamma} e^{-i c \gamma}
$$

where $\tau=\tau(E):=2 \sqrt{m^{2}-E^{2}}$ and $\kappa:=\kappa(E):=\gamma E / \sqrt{m^{2}-E^{2}}$. Then $E \in$ $(-m, m)$ is an eigenvalue of $\widetilde{d}_{\mathcal{L}}^{\infty, B}$ in the \pm -sector of the Parity $\mathscr{G}$ Exchange operator $P \mathcal{E}$ iff $F_{ \pm}(E)=e^{i \gamma \log \widetilde{B}}=B^{i \gamma}$.

An equivalent way of stating the eigenvalue condition is that $A_{ \pm}(E):=$ $\operatorname{Arg}\left(F_{ \pm}(E)\right)=i \gamma \log B \bmod 2 \pi$, where $\operatorname{Arg}(\zeta) \in(-\pi, \pi]$ denotes the principal value of the argument of $\zeta \in \mathbb{C} \backslash 0$. Graphical analysis shows that for small $\gamma$ and given $B>0, \widetilde{d}_{\mathcal{L}}^{\infty, B}$ will have infinitely many eigenvalues $E_{0}^{ \pm}(B)<E_{1}^{ \pm}(B)<\cdots$ in $(-m, m)$ accumulating at $m$, see figure 1 below, for an illustration for $\gamma=0.5$. The eigenvalues in either sector are monotonically decreasing in $B$, and the lowest eigenvalue $E_{0}^{+}(B)$ will, for a certain critical value $B_{c}$ of the field, be absorbed into lower continuous spectrum $(-\infty,-m]$, at which point $E_{0}^{-}(B)$ will become the new lowest eigenvalue, and the whole process will repeat itself, periodically in $\log B$ with period $2 \pi / \gamma$. As a consequence of norm-resolvent convergence, the same phenomenon will occur for $d_{\mathcal{L}}^{B}$ if $B$ is sufficiently large, the periodicity becoming approximate. We note that in reference [3] it was recently shown, by a variational argument involving a min-max characterisation of the ground state of Dirac operators in the spectral gap $(-m, m)$ and comparison with $d_{\mathcal{L}}^{B}$, that the ground state of $D^{B}$ enters the negative continuous spectrum for a certain $B_{c}=B_{c}(\gamma)$. This left open the question of whether there remained other eigenvalues. Our result answers this for $d_{\mathcal{L}}^{B}$ when $B$ is sufficiently large, and also for $D^{B}$ in the limit of $\gamma \sqrt{B} \rightarrow 0$.

Concerning the critical values of $B$, one can use Stirling's formula to show that

$$
\lim _{E \rightarrow-m} F_{ \pm}(E)=\mp \frac{\Gamma(1-2 i \gamma)}{\Gamma(1+2 i \gamma)}\left(\frac{2 \gamma m}{e^{c / 2}}\right)^{2 i \gamma}
$$

The critical values $B_{c}$ of the magnetic field for which $E_{0}^{ \pm}\left(B_{c}\right)=-m$ will have to satisfy $B_{c}^{i \gamma}=$ Right Hand Side of (3.2). We note that if $B_{c}(\gamma)$ is the first critical $B>1$, then $\lim _{\gamma \rightarrow 0} \gamma \log B_{c}=\pi$, confirming a result of [3]; (3.2) allows us to derive an asymptotic expansion of $\gamma B_{c}(\gamma)$ for small $\gamma$.

Acknowledgement. This research was supported by a grant from Alliance: Franco-British Partnership Programme 2006 and 2007, project Number PN 06.015. 


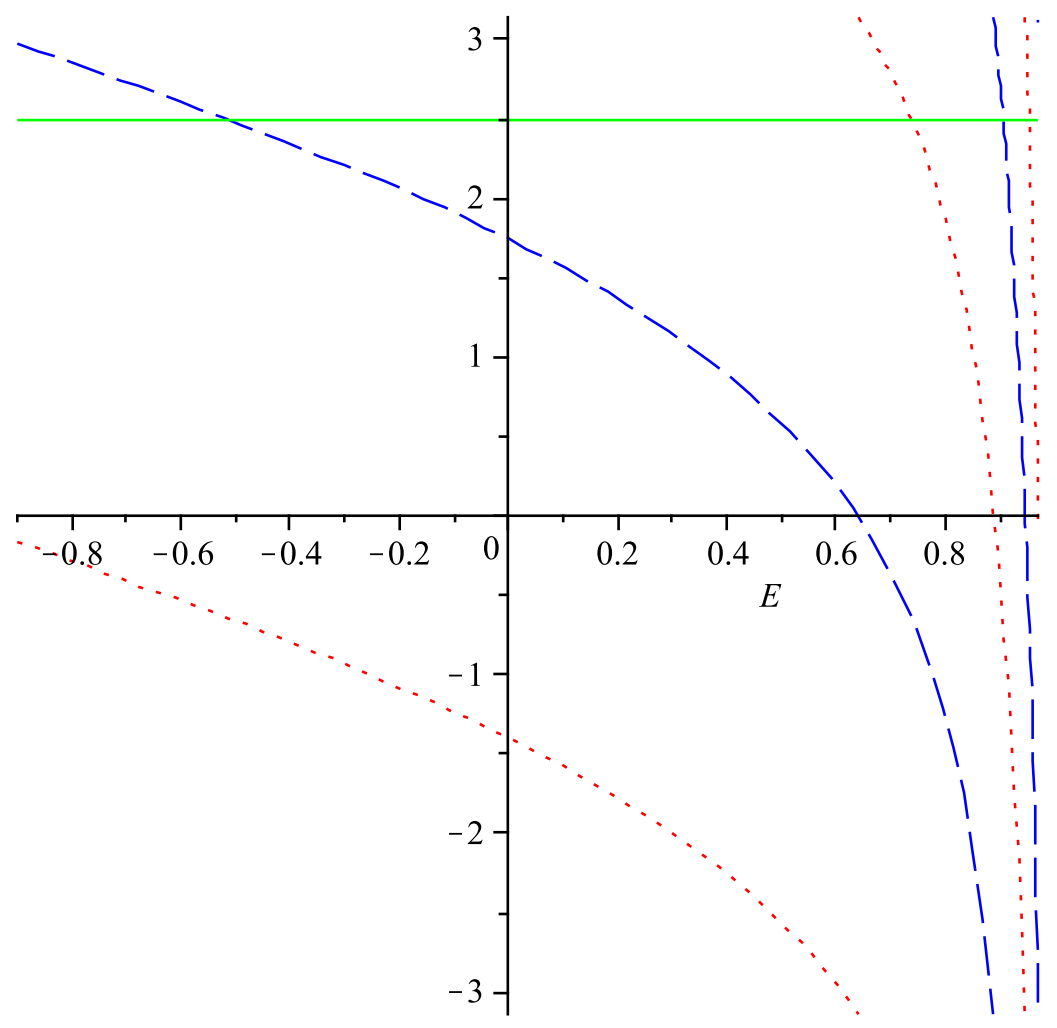

Figure 1. Graphs of $A_{+}(E)$ (dots), $A_{-}(E)$ (dashes) and $\gamma \log B=2.5 \bmod 2 \pi$ (solid), $\gamma=0.5$

\section{References}

1. Briet, Ph., Brummelhuis, R., and Duclos, P.: Effective Hamiltonians for relativistic hydrogen in a strong homogeneous magnetic field, in preparation.

2. Brummelhuis, R. , Duclos, P.: Effective hamiltonians for atoms in very strong magnetic fields, J. Math. Phys. 47, 032103 (2006).

3. Dolbeault, J., Esteban, M., Loss, M.: Relativistic Hydrogenic Atoms in Strong Magnetic Fields Ann. H. Poincare 8, 749 (2007). 